\title{
ON SHARPNESS OF SOME INTEGRAL INEQUALITIES AND AN INTEGRAL EQUATION OF VOLTERRA TYPE
}

\author{
Josip PEČARIĆ, IVAN PERIĆ AND LARS-ERIK PERSSON
}

\begin{abstract}
The sharpness of some recent integral inequalities is discussed and the corresponding extremal functions are pointed out. It is also proved that the cases of equality can equivalently be obtained by solving an integral equation of Volterra type with discontinous kernel $\chi_{A}(t)$. This integral equation of independent interest is solved for every measurable set $A$ on $(a, b),-\infty<$ $a<b<\infty$.
\end{abstract} function.

Mathematics subject classification (2000): 26D15, 27D07.

Key words and phrases: integral inequalities, integral equations, decreasing function, increasing

\section{REFERENCES}

[1] S. BARZA, J. PeČARIĆ, L. E. Persson, Reversed Hölder type inequalities for monotone functions of several variables, Math. Nachr. 186 (1997), 67-80.

[2] J. Bergh, V. I. Burenkov, L. E. Persson, Best constants in reversed Hardy's inequalities for quasimonotone functions, Acta Sci. Math. (Szeged) 59 (1994), 221-239.

[3] J. BERGH, V. I. BURENKOV, L. E. PERSSON, On some sharp reversed Hölder and Hardy type inequalities, Math. Nachr. 169 (1994), 19-29.

[4] V. I. BuRENKov, On the best constant in Hardy's inequality for $0<p<1$. Trudy Mat. Instit. im Steklova 194 (1992), 58-62 (in Russian).

[5] R. J. Bushel, W. OKRASINSKI, Volterra equations with convolution kernel, J. London Math. Soc. 41 (1990), 503-510.

[6] A GARCIA DEL AMO, On reverse Hardy's inequality, Collectanea Math. 44 (1993), 115-123.

[7] G. H. HaRdy, J. E. LitTlewood, G.Pólya, Inequalities, Cambridge Univ. Press 1952.

[8] H. HeINIG, L. MALIGRANDA, Weighted inequalities for monotone and concave functions, Studia Math. 116 (2) (1995), 133-165.

[9] G. G. LORENTZ, Some new functional spaces, Ann. of Math. 51 (1950), 37-55.

[10] W. G. MAZJA, Einbettungssätze für Sobolewsche Raume I, Teubner, Leipzig, 1979.

[11] J. PeČARIĆ, L. E. PERSSON, On Bergh's inequality for quasi-monotone functions, J. Math. Anal. Appl. 195 (1995), 393-400.

[12] J. PEČARIĆ, I. PERIĆ, L. E. PERSSON, A multidimensional integral inequality for monotone functions of several variables, Acta Sci. Math (Szeged) 62 (1996), 407-412.

[13] J. PeČARIĆ, I. PERIĆ, L. E. PERSSON, Integral inequalities for monotone functions, J. Math. Anal. Appl. 215 (1997), 235-251.

[14] E. SteIn, G. WeISs, Introduction to Fourier Analysis on Euclidean Spaces, Princeton Univ. Press, Princeton, New Jersey 1971.

[15] W. WALtER, V. WeCKESSER, An integral inequality of convolution type, Acquationes Math. 46 (1993), 212-219. 